\title{
Highly sensitive contact pressure measurements using FBG patch in endotracheal tube cuff
}

\author{
R. Correia ${ }^{\text {a }}$, O.R. Blackman ${ }^{\mathrm{a}}$, F.U. Hernandez, S. Korposh*a, S.P. Morgan ${ }^{\mathrm{a}}$, B. R. Hayes-Gill ${ }^{\mathrm{a}}$, S.W.

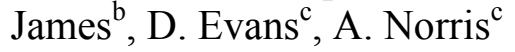 \\ ${ }^{a}$ Advanced Optics Group, Faculty of Engineering, University of Nottingham, Nottinghamshire, NG7 \\ 2RD, UK; ${ }^{\text {b }}$ Engineering Photonics, Cranfield University, Bedfordshire, MK43 0AL, UK.; \\ 'Department of Anaesthesia, Queen's Medical Centre, Nottingham, NG7 2UH, UK
}

\begin{abstract}
A method for measuring the contact pressure between an endotracheal tube cuff and the trachea was designed and developed by using a fibre Bragg grating (FBG) based optical fibre sensor. The FBG sensor is encased in an epoxy based UV-cured cuboid patch and transduces the transversely loaded pressure into an axial strain that induces wavelength shift of the Bragg reflection. The polymer patch was created by using a PTFE based mould and increases tensile strength and sensitivity of the bare fibre FBG to pressure to $2.10 \times 10-2 \mathrm{~nm} / \mathrm{kPa}$. The characteristics of the FBG patch allow for continuous measurement of contact pressure. The measurement of contact pressure was demonstrated by the use of a 3D printed model of a human trachea. The influence of temperature on the measurements is reduced significantly by the use of a second FBG sensor patch that is not in contact with the trachea. Intracuff pressure measurements performed using a commercial manometer agreed well with the FBG contact pressure measurements.
\end{abstract}

Keywords: Fibre Bragg Grating (FBG), intracuff pressure, contact pressure, endotracheal tube (ETT).

\section{INTRODUCTION}

Endotracheal tubes (ETTs) are used in medical care to establish and maintain an airway to allow the delivery of oxygen or an anaesthesic to the lungs through the trachea. This is particularly useful in cases where patients are undergoing general anaesthesia and require intubation ${ }^{1}$. ETT cuffs are used to obstruct the air path between the trachea and the ETT' ${ }^{2}$. The cuff is a balloon which, when inflated, centres the ETT in the trachea. The centred ETT cuff prevents trauma of the mucosal lining, air leaks to the atmosphere and oropharyngeal secretions into the lungs ${ }^{2}$.

The presence of the cuffed ETT in the trachea for prolonged periods of time can lead to significant clinical problems. Firstly, it has been demonstrated that if cuff pressures are too high they damage the mucosal lining of the trachea, mainly through the applied contact pressure reducing microcirculatory flow, and therefore oxygen supply, to the epithelial cells. This deterioration of tracheal mucosal blood flow is a critical factor in tracheal morbidity associated with intubation and development of post intubation tracheal stenosis ${ }^{1}$. Therefore, it is important to pay attention to cuff pressure. However, lack of routine use of manometers and the difficulty of their deployment in the trachea prevents this. Furthermore, due to the rings of cartilage in the trachea, the intracuff pressure may not be representative of the contact pressure at different positions in the traches. This opens up the need for a routine way to measure contact pressure when performing endotracheal intubation.

Fibre Bragg grating (FBG) based optical fibre sensors (OFS) have been adapted to pressure sensing with a high sensitivity ${ }^{3,4}$. FBG sensors have the capability to measure over the quoted intracuff pressure range from $20 \mathrm{~cm}_{2} \mathrm{O}$ up to $50 \mathrm{~cm} \mathrm{H}_{2} \mathrm{O}^{5}$. We have recently reported ${ }^{3}$ a contact pressure sensor using FBGs that were glued to the outside wall of the cuff and provided some evidence of a correlation between intra cuff pressure and contact pressure. In this work we report an improvement of the sensitivity and accuracy of the FBG sensor via its incorporation into a patch ${ }^{4}$ that transforms contact pressure into strain and avoids bending of the FBG. To further improve the robustness of the measurements, a second FBG is been used to provide temperature reference measurements. The FBG patch was attached to the cuff and tested using a 3D model of the human trachea.

Sixth European Workshop on Optical Fibre Sensors, edited by Elfed Lewis, Proc. of SPIE Vol. 9916, $99161 \mathrm{~F} \cdot$ ? 2016 SPIE · CCC code: $0277-786 \mathrm{X} / 16 / \$ 18 \cdot$ doi: $10.1117 / 12.2236859$ 


\section{EXPERIMENT}

Two FBGs with lengths of $2 \mathrm{~mm}$ were fabricated in the core of an $\mathrm{H}_{2}$ loaded optical fibre (Fibrecore SM28) using a phase mask to provide Bragg wavelengths of $1544 \mathrm{~nm}$ and $1549 \mathrm{~nm}$, respectively. To increase the sensitivity of the FBG to the contact pressure, the FBG was incorporated into the patch using a UV curable epoxy and PTFE mold as shown in Figure 1a. A small amount of epoxy was deposited onto the top of the FBG and was exposed to a 10 Watt UV flashlight operating at a wavelength of $365 \mathrm{~nm}$ for 10 minutes. The FBG embedded into the patch was calibrated for transverse load measurements by placing a known weight on the top of the patch and recording the reflection spectrum using a SmartScan FBG interrogator. In order to calibrate the pressure response of the sensor, the mass on the patch was increased in steps $(25 \mathrm{~g})$ and then decreased back to the original value. This was done in order to ensure that the polymer material was sufficiently elastic to ensure a similar expansion when loaded and unloaded. Additionally, the mass was converted to pressure in $\mathrm{kPa}$ using $\mathrm{P}=\mathrm{mg} / \mathrm{A} \times 10^{-3}$, where $\mathrm{m}$ is the measured mass on the patch in $\mathrm{kg} ; \mathrm{g}=9.81 \mathrm{~m} / \mathrm{s}^{2}$ is the assumed effective gravitational acceleration of earth; and $\mathrm{A}$ is the area of the patch over which the force is exerted. The patch was taken through two cycles of loading and unloading. A total of 81 data points with a corresponding 10 second $\log$ of the reflected Bragg wavelength value was recorded. The mean wavelength for each log was then taken as being representative of each mass value.

In order to analyse the performance of the FBG in the patch in measuring contact pressure, a trachea model was designed and manufactured. One of the patches containing an FBG was attached to the cuff (Fig. 1b) while the other was left unattached to provide a temperature reference (Fig. 1b). The separation between the two FBGs was ca. $9 \mathrm{~mm}$. The ETT with the attached FBG sensors was then inserted into the 3D model (Fig. 1c) of the human trachea in order to measure contact pressure. Intracuff pressure was measured using a digital manometer (EXTECH, HD750).

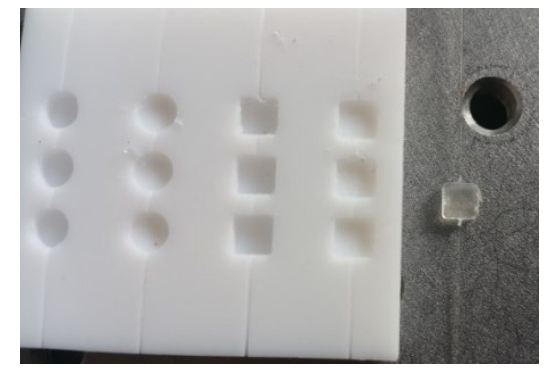

(a)

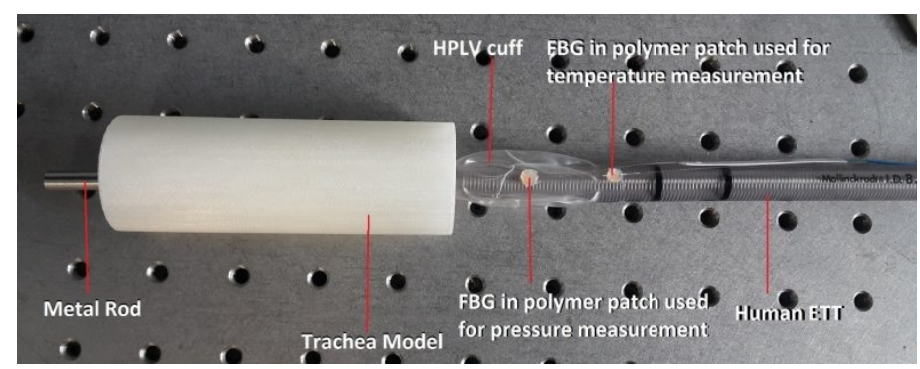

(b)

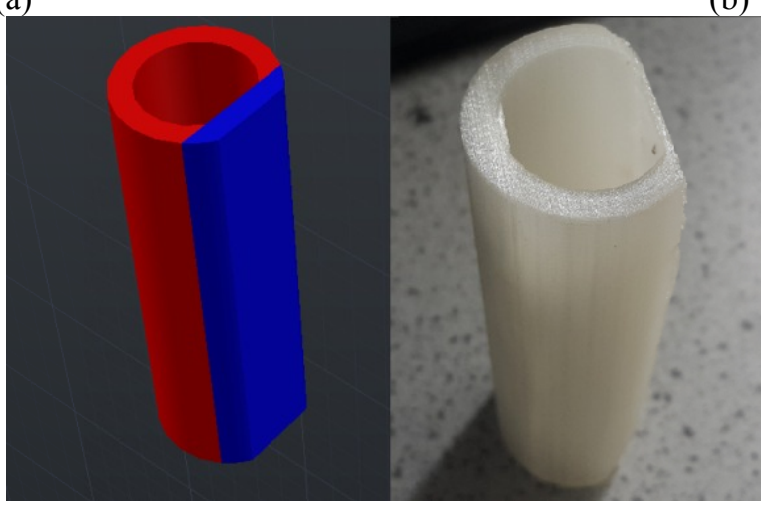

(c)

Figure 1. (a) PTFE mold used to embed the FBG sensor into the epoxy patch; (b) photograph of the ETT containing FBG sensors and 3D printed model of the human traches; and (c) 3D design (left) and printed (right) models of the human trachea model without rings of cartilage; the passage size of the trachea in Figures 1c is $3 \mathrm{~cm}$.

\section{RESULTS AND DISCUSSIONS}

Figure 2a shows the change of the Bragg wavelength with time at the increase and decrease of the load. Maximum Bragg wavelength shifts of $0.0582 \mathrm{~nm}$, corresponding to a pressure of $59.4731 \mathrm{kPa}$, and $0.0532 \mathrm{~nm}$ for a pressure of 61.9256 $\mathrm{kPa}$, were observed while loading and unloading, respectively. The difference is most likely a result of the ability of the patch to relax. The data points were then broken down into four sections, two loading and two unloading, with each 
section beginning and ending at a turning point on the graph. A straight line of best fit was found for each section and the gradient of each line was taken to determine the sensitivity. The average value of sensitivity to pressure of the FBG in the polymer patch was found to be around $1 \times 10^{-2} \mathrm{~nm} / \mathrm{kPa}$.

Figure $2 \mathrm{~b}$ shows the change of the contact pressure measured by the FBG and the intracuff pressure measured by the manometer at the cuff inflation. As can be seen, the contact pressure values are very similar to the intracuff pressure. Interesting results were observed around 10 seconds, when the cuff starts to be inflated. The intracuff pressure starts to increase as expected while the contact pressure does not change until 10 seconds later, when the cuff touches the wall of the trachea model. These results indicate that the FBG sensor can provide extremely valuable information about the time when the cuff comes into contact with the wall of the trachea. This is important as it could potentially be used to indicate when the cuff has obtained a tight seal, avoiding over-inflating the cuff.

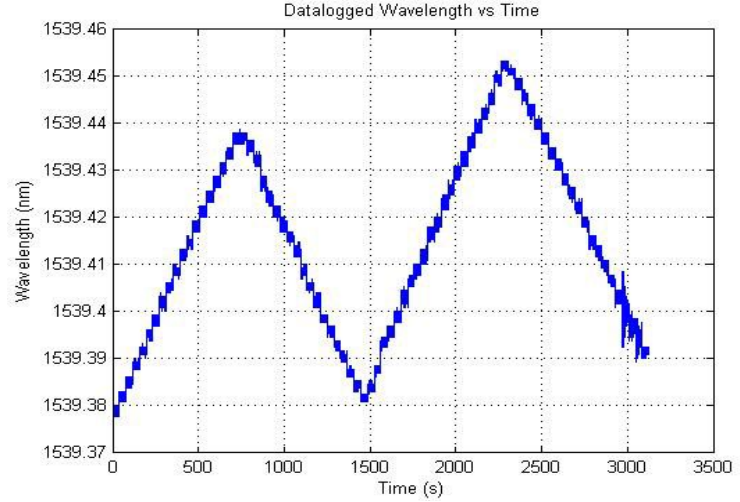

(a)

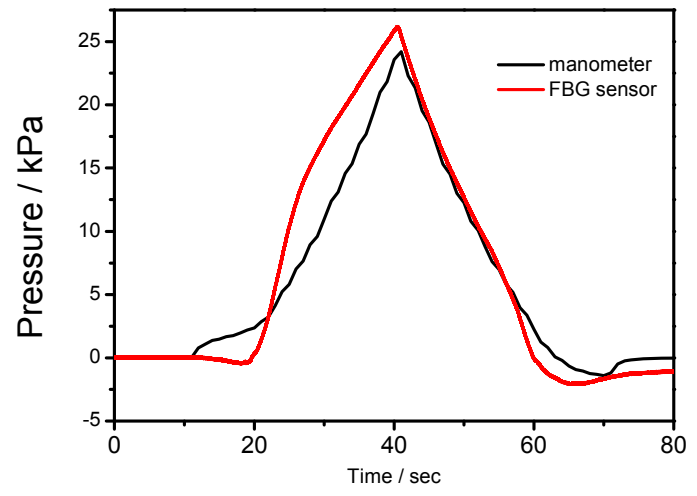

(b)

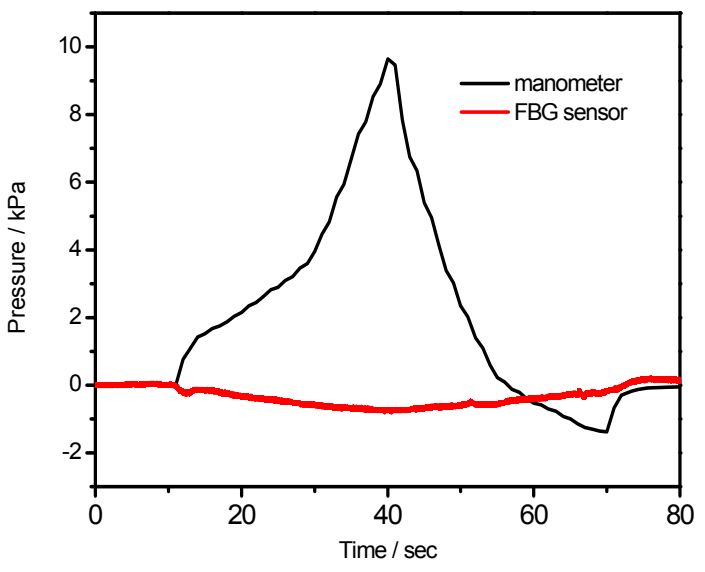

(c)

Figure 2. (a) Dynamic wavelength shift of the FBG sensor embedded into the patch at the increase and decrease of the load; (b) Contact pressure (red line) measured using FBG sensor embedded into the patch and intracuff pressure (black line) measured using a manometer at the inflation of the ETT cuff inserted into the 3D printed model of the trachea; and (c) Contact pressure (red line) measured using FBG sensor embedded into the patch and intracuff pressure (black line) measure using manometer at the inflation of the ETT cuff outside of the 3D printed model of the trachea.

In order to test this further, the ETT with embedded FBG sensors was tested outside of the 3D printed model of the trachea, Figure 2c. As can be seen from the figure 2c, the intracuff pressure increases as the cuff is inflated, while the change in the contact pressure (FBG response) is negligible. The total reflected Bragg wavelength shift outside the trachea is much smaller at $0.0270 \mathrm{~nm}(1.27 \mathrm{kPa})$ as compared to the measurements inside the trachea of $0.4879 \mathrm{~nm}$ $(23.014 \mathrm{kPa})$ for the flat surface and $0.6231 \mathrm{~nm}(29.392 \mathrm{kPa})$ for the curved surface (as shown in fig. 1c) when the same volume of air is used. Therefore it is seen that the total FBG pressure is highest when in contact with the curved surface possibly because the curvature of the model causes the central section of the patch to be pressed into this. The negligible 
1.27 kPa FBG pressure response outside the cuff could be attributed to a strain being exerted on the fibre from the stretching of the cuff. This could be resolved by a modification of the means for attachment of the patch in such a ways that it is not exposed to the stretching of the cuff. Nevertheless these results demonstrate that the response of the FBG sensor embedded into the patch is dominated by the contact pressure.

\section{CONCLUSIONS}

A method for measuring the contact pressure between an endotracheal tube cuff and the trachea was designed and developed by using a fibre Bragg grating (FBG) based optical fibre sensor embedded into a patch. The results obtained give an optimistic view of future implementation of a non-intrusive in situ sensor for contact pressure during endotracheal intubation. The pressure sensitivity of the FBG patch was found to be around $2.10 \times 10-2 \mathrm{~nm} / \mathrm{kPa}$. The FBG patch pressure sensor introduces a more useful measurement method because there is currently no other method to measure contact pressure on the tracheal wall. Intracuff measurements would therefore need to be used to calibrate the FBG patch sensor contact pressure. The discrepancy between the pressure measured by the FBG patch and the intracuff pressure is taken to be the contact pressure. From observation of the FBG patch when outside the trachea model it is seen that only a small wavelength shift occurs further indicating that it is the contact pressure, rather than inflation of the tube, that is being measured. However a greater contact pressure was measured on the curved surface compared to that measured on the flat surface.

The best placement for the FBG patch was found to be at a position halfway along the cuff. However there is some merit in using an array of sensors to ensure that multiple points of contact are found. This way, the FBG patch which reaches the maximum contact pressure first could be taken as the limit. Temperature effects can be removed by having a second FBG patch sensor which is placed on the ETT but not on the cuff. Any wavelength change of this separate temperature FBG patch could be subtracted from the pressure wavelength. This removes the need to undertake a temperature calibration for any of the FBG patches. PTFE was found to be a suitable polymer patch mold material, with the NOA65 UV-cured polymer being used as a suitable patch material. This polymer is also suitable for manual recoating of the bare fibre, increasing both the tensile strength of the bare fibre and sensitivity to pressure.

\section{ACKNOWLEDGMENT}

The authors want to thank the National Institute for Health Research Invention for Innovation (NIHR i4i) Programme (Grant Reference Number: II-LA-0813-20008) for funding. The views expressed are those of the authors and not necessarily those of the NHS, the NIHR or the Department of Health. FUH acknowledges the support of the National Council of Science and Technology of Mexico (CONACyT) and the Ministry of Public Education in Mexico (DGRISEP) for a scholarship.

\section{REFERENCES}

[1] Seegobin, R. D. and Van Hasselt, G. L., "Endotracheal cuff pressure and tracheal mucosal blood flow: endoscopic study of effects of four large volume cuffs", BMJ 288, 965-968 (1984).

[2] R. D. Seegobin and G. L. van Hasselt, "Aspiration beyond endotracheal cuffs," Canadian Anaesthetists' Society Journal, vol. 33, no. 3, pp. 273-279, 1986.

[3] Hernandez, F.U., Correia, R., Korposh, S., Morgan, S.P., Hayes-Gill, B. R., James, S.W., Evans, D., Norris, A., "Measurements of endotracheal tube cuff contact pressure using fibre Bragg gratings", Proc. SPIE 9634, 24th International Conference on Optical Fibre Sensors, 963435 (September 28, 2015); doi:10.1117/12.2195118.

[4] Correia, R., Chehura, E., Li, J., James, S. W., Tasam, R. P., "Enhanced sensitivity fibre Bragg grating (FBG) load sensor", Measurements Science and Technolog, 21(9), 094006, 2010.

[5] Goethals, P., Chaobal, H., Reynaerts, D., and Schaner, D., "A novel airway device with tactile sensing capabilities for verifying correct endotracheal tube placement”, J Clin Monit Comput 28(2), 179-185 (2014). 
2016-05-31

Highly sensitive contact pressure measurements using FBG patch in endotracheal tube cuff

Correia, Ricardo N.

SPIE

Correia et al. (2016) Highly sensitive contact pressure measurements using FBG patch in endotracheal tube cuff, 6th European Workshop on Optical Fibre Sensors (EWOFS'2016), Limerick, Ireland, 30-31 May 2016 http://dx.doi.org/10.1117/12.2236859

Downloaded from Cranfield Library Services E-Repository 